\title{
Atomic migration studies with X-ray photon correlation spectroscopy
}

\author{
Markus Stana $^{1, \mathrm{a}}$, Manuel Ross ${ }^{1, \mathrm{~b}}$, and Bogdan Sepiol ${ }^{1, \mathrm{c}}$ \\ Universität Wien, Faculty of Physics, Dynamics of Condensed Systems, Boltzmanngasse 5, 1090 \\ Wien, Austria \\ aamarkus.stana@univie.ac.at, ${ }^{b}$ manuel.ross@univie.ac.at, cbogdan.sepiol@univie.ac.at
}

Keywords: chemical diffusion, diffusion, diffuse scattering, dynamic light scattering, Ni-Pt alloy, shortrange order, Si-Ge, synchrotron radiation, XPCS

\begin{abstract}
The new technique of atomic-scale X-ray Photon Correlation Spectroscopy (aXPCS) makes use of a coherent X-ray beam to study the dynamics of various processes in condensed matter systems. Particularly atomistic migration mechanisms are still far from being understood in most of intermetallic alloys and in amorphous systems. Special emphasis must be given to the opportunity to measure atomistic diffusion at relatively low temperatures where such measurements were far out of reach with previously established methods. The importance of short-range order is demonstrated on the basis of Monte Carlo simulations.
\end{abstract}

\section{Introduction}

Numerous properties of materials can be attributed to the movement of single atoms. Understanding the processes involved in atomic transport is therefore of great importance for improving both the fabrication and the stability of materials. The majority of experimental techniques applied to measure diffusion are only sensitive to macroscopic scales corresponding to thousands of atomic jumps. Just a few methods are sensitive to movements on the atomic scale, which means that they are able to resolve the migration of single atoms in time and in space. Almost all of these techniques are, however, sensitive only to fast diffusion on the order of $10^{-14} \mathrm{~m}^{2} \mathrm{~s}^{-1}$ or faster and limited to a restricted number of suitable isotopes. Due to these limiting factors it is very difficult or even impossible to apply these methods for studying metastable systems like glasses or nanocrystalline materials on the atomic scale. The advent of modern synchrotron sources paved the way for a new experimental technique which is not subject to these restrictions.

The following review chapter is an introduction to this new method for studying dynamics in solid state materials called atomic-scale X-ray photon correlation spectroscopy (aXPCS). In a nutshell, it relies on the fact that coherent X-rays scattered at a particular arrangement of atoms in a sample produce a corresponding intensity pattern. Correlating several series of these patterns collected for certain scattering angles over a given amount of time yields information that allows to establish a model of atomistic hopping mechanisms in the material. In that way, dynamics on the atomic scale in crystalline as well as in amorphous matter are experimentally accessible. The new technique at hand allows to study diffusion in intermetallic phases at low temperatures where otherwise only data from the tracer diffusion method is available, yet it comprises spacial resolution of jumps on the atomic scale. Information on the atomic diffusion mechanisms of amorphous systems can be experimentally obtained well below the glass transition temperature, where the question of dynamics is far from being solved.

This review is be organized in the following way: the first chapter is devoted to the theoretical foundations of the method. We shall endeavor to present a short and compact but self-contained description of the method including in particular application in the atomistic migration in intermetallics and in amorphous materials. The coherent method of aXPCS contrary to many incoherent methods for studying diffusion relies on the knowledge of the short-range order (SRO) of the system under investigation. Special attention is given to this special feature of this coherent method. 
In a short section the experimental setup and sample environment is be described. This is followed by a selection of systems measurable by aXPCS. The influence of short-range order is demonstrated starting with the simple case of self-diffusion, where SRO is governed by a repulsive force between components of the alloy. This is done on the basis of Monte Carlo (MC) simulations in nickel-platinum alloys and compared with experimental results. This approach is then extended to a wider range of Ni-Pt compositions. Short-range order parameters can be controlled in their pure state using MC techniques, i.e. without any disturbance by a displacement scattering. Moreover, the chemical diffusion coefficient is simulated by means of the MC method and compare it to the coefficient obtained from an aXPCS computer experiment, proving the validity of this method in a wide range of stoichiometries. This approach was, to our knowledge, not used up to now. A section on diffusion in semiconductors presents the first aXPCS measurement of a multi-exponential autocorrelation function decay.

An outlook into the bright future of the aXPCS technique closes this review.

\section{Theoretical background}

Let us consider a lattice that is built up by two types of atoms called $A$ and $B$. We can define a state function, which tells us what kind of atom occupies a particular lattice site $r$,

$$
\sigma(\mathbf{r})= \begin{cases}0 & \text { if } A \text { at } \mathbf{r} \\ 1 & \text { if } B \text { at } \mathbf{r} .\end{cases}
$$

The state function $\sigma(\mathbf{r}, t)$ becomes time dependent if diffusion takes place and it can be used to define a correlation function:

$$
G(\Delta \mathbf{r}, \Delta t)=\langle\sigma(\mathbf{r}, t) \sigma(\mathbf{r}+\Delta \mathbf{r}, t+\Delta t)\rangle_{\mathbf{r}, t}
$$

This autocorrelation function is called Van Hove correlation function [1]. Here the brackets $\langle\ldots\rangle_{\mathbf{r}, t}$ indicate an ensemble average over space and time but these subscripts will be omitted in the following text, whereas $\Delta t$ is a time-lag within the whole event. $G(\Delta \mathbf{r}, \Delta t)$ will decay within a specified decaytime depending on the mobilities of atoms in the system. If we know the function $G(\Delta \mathbf{r}, \Delta t)$, we have the full information about the diffusing system. The Van Hove correlation function is also called pair correlation function.

We can carry out a scattering experiment on a finite number of discrete scatterers using a coherent light source. Then $\sigma\left(\mathbf{r}_{j}\right)=\sigma_{j}$ and the electric field vector is given by

$$
E(\mathbf{q}) \propto \sum_{j} f_{j} \mathrm{e}^{\mathrm{i} \mathbf{q} \cdot \mathbf{r}_{j}}
$$

where $f_{j}$ is the scattering length of the atom at $r_{j}$ and $\mathbf{q}=\mathbf{k}_{\text {out }}-\mathbf{k}_{\text {in }}$ is the scattering vector. If the scattering length is proportional to the state function in real space $f_{j}=\lambda \sigma_{j}$ (in the case of Eq. 1 the scattering length of atoms of type $A$ would therefore be 0 and only atoms of type $B$ would act as scatterers), this can be written as a Fourier transform

$$
E(\mathbf{q}) \propto \mathscr{F}\left(\lambda \sigma_{j}\right) .
$$

The electric field amplitude can be used analogously to the state function to define a temporal autocorrelation function:

$$
g^{(1)}(\mathbf{q}, \Delta t)=\frac{\left\langle E(\mathbf{q}, t) E^{*}(\mathbf{q}, t+\Delta t)\right\rangle}{\left\langle E(\mathbf{q}, t) E^{*}(\mathbf{q}, t)\right\rangle}
$$

The numerator of Eq. 5 can be written using a sum similar to Eq. 3 as:

$$
\sum_{i, j}\left\langle f_{i} f_{j} \exp \left\{\mathbf{i q} \cdot\left[\mathbf{r}_{i}(t)-\mathbf{r}_{j}(t+\Delta t)\right]\right\}\right\rangle \equiv \mathscr{I}(\mathbf{q}, \Delta t)
$$


and is referred to in the neutron scattering literature as the intermediate scattering function (temporal Fourier transform of the Van Hove correlation function) [2]. In this text, when using the term intermediate scattering function we refer to the normalized function $g^{(1)}(\mathbf{q}, \Delta t)$, see Eq. 5 .

Measuring the electric field amplitudes as a function of time for a certain vector $q$ in reciprocal space would allow to study diffusion in a similar way as to follow the development of a state function in real space. However, the loss of information due to the phase problem makes direct observation of the amplitudes impossible in small time intervals.

What is possible, however, is to measure the scattering intensities $I(\mathbf{q})$. We use this quantity, again time dependent, and define a normalized autocorrelation function similar to Eq. 5, which we call the intensity autocorrelation function:

$$
g^{(2)}(\mathbf{q}, \Delta t)=\frac{\langle I(\mathbf{q}, t) I(\mathbf{q}, t+\Delta t)\rangle}{\langle I(\mathbf{q}, t)\rangle^{2}}
$$

Using the fact that the intensity is the square of the amplitude of the electromagnetic field $I(\mathbf{q}, t)=$ $E(\mathbf{q}, t) E^{*}(\mathbf{q}, t)$ and that the fields of individual sources are not influencing each other, we can expand Eq. 7:

$$
g^{(2)}(\mathbf{q}, \Delta t)=\frac{\left\langle E(\mathbf{q}, t) E^{*}(\mathbf{q}, t) E(\mathbf{q}, t+\Delta t) E^{*}(\mathbf{q}, t+\Delta t)\right\rangle}{\left\langle E(\mathbf{q}, t) E^{*}(\mathbf{q}, t)\right\rangle^{2}}
$$

As Isserlis' theorem holds for Gaussian-like scatterers [3], i.e. if the statistics of the electric field are Gaussian of zero mean, only the second moments have to be considered and correlation functions of the right-hand side of Eq. 8 can be expressed as a sum of all the possible products of two-time field correlations:

$$
\begin{aligned}
& \left\langle E(t) E^{*}(t) E(t+\Delta t) E^{*}(t+\Delta t)\right\rangle= \\
& \left\langle E(t) E^{*}(t)\right\rangle\left\langle E(t+\Delta t) E^{*}(t+\Delta t)\right\rangle+\langle E(t) E(t+\Delta t)\rangle\left\langle E^{*}(t+\Delta t) E^{*}(t)\right\rangle+ \\
& \left\langle E(t) E^{*}(t+\Delta t)\right\rangle\left\langle E(t+\Delta t) E^{*}(t)\right\rangle= \\
& \langle I(\mathbf{q}, t)\rangle^{2}+\left\langle\left|E_{0}\right| \mathrm{e}^{\mathrm{i} \phi}\left|E_{0}\right| \mathrm{e}^{\mathrm{i} \phi^{\prime}}\right\rangle\left\langle\left|E_{0}\right| \mathrm{e}^{-\mathrm{i} \phi^{\prime}}\left|E_{0}\right| \mathrm{e}^{-\mathrm{i} \phi}\right\rangle+ \\
& \left\langle E(t) E^{*}(t+\Delta t)\right\rangle\left\langle E(t+\Delta t) E^{*}(t)\right\rangle
\end{aligned}
$$

The time average of a very fast oscillating phase $\left(\omega_{0} \approx 10^{-15} \mathrm{~s}^{-1}\right)$ at a certain point in space vanishes, which means that $\left\langle\exp \left(2 \mathrm{i} \omega_{0} t\right)\right\rangle=0$. Therefore we are left with:

$$
g^{(2)}(\mathbf{q}, \Delta t)=1+\left(\frac{\left|\left\langle E(\mathbf{q}, t) E^{*}(\mathbf{q}, t+\Delta t)\right\rangle\right|}{\left\langle E(\mathbf{q}, t) E^{*}(\mathbf{q}, t)\right\rangle^{2}}\right)^{2}=1+\left|g^{(1)}(\mathbf{q}, \Delta t)\right|^{2}
$$

This equation is well known as the Siegert relation [4] and it was proven that it is fulfilled even under harsh experimental conditions [5]. Utilizing this relation an intermediate scattering function $g^{(1)}(\mathbf{q}, \Delta t)$ given by a theoretical model can be directly compared to the experimental scattering results. It is possible to measure $g^{(2)}(\mathbf{q}, \Delta t)$ over a wide dynamical range which is practically only limited by the number of coherent photons scattered by the sample. Using typical commercial correlators, $\Delta t$ can range from nanoseconds to hours, covering a range of twelve orders of magnitude. This is the main reason why the method of dynamic light scattering found extensive propagation in colloids dynamic research $[6,7]$.

In order to find a way to derive the intermediate scattering function from fundamental considerations we introduce a probability density $P(\mathbf{r}, t)$. It gives the probability of finding an atom of type $B$ at a certain position at a certain time and therefore equals the ensemble average of the state function:

$$
P(\mathbf{r}, t)=\langle\sigma(\mathbf{r}, t)\rangle
$$

Let us consider a Bravais lattice where the jump probability depends on the jump length $\Delta \mathbf{r}$ but not on the site occupied by the atom and only on the present state of the system (Markov or memoryless 
process). The probability for an atom to be at a certain position $\mathbf{r}$ at time $t+\Delta t$ is given by the probability of being at the same position as at time $t$ without moving away, plus the probability for particles that were at the neighboring site $\mathbf{r}-\Delta \mathbf{r}$ in a distance $\Delta \mathbf{r}$ and jump into $\mathbf{r}$ during the time interval $\Delta t$,

$$
P(\mathbf{r}, t+\Delta t)=P(\mathbf{r}, t)\left(1-\frac{\Delta t}{\tau_{0}} \sum_{\Delta \mathbf{r}} p(\Delta \mathbf{r})\right)+P(\mathbf{r}-\Delta \mathbf{r}, t) \frac{\Delta t}{\tau_{0}} \sum_{\Delta \mathbf{r}} p(\Delta \mathbf{r}),
$$

with $\tau_{0}$ being the time an atom resides at a certain position in the lattice and $p(\Delta \mathbf{r})$ being the probability for an atom to instantaneously jump to another site a distance $\Delta \mathbf{r}$ away. This can be reordered,

$$
\lim _{\Delta t \rightarrow 0} \frac{P(\mathbf{r}, t+\Delta t)-P(\mathbf{r}, t)}{\Delta t}=-P(\mathbf{r}, t) \frac{1}{\tau_{0}} \sum_{\Delta \mathbf{r}} p(\Delta \mathbf{r})+P(\mathbf{r}-\Delta \mathbf{r}, t) \frac{1}{\tau_{0}} \sum_{\Delta \mathbf{r}} p(\Delta \mathbf{r}),
$$

and in the limit of very small time intervals $\Delta t$ it can be rewritten in the form of a differential equation (called rate equation)

$$
\frac{\partial P(\mathbf{r}, t)}{\partial t}=\frac{1}{\tau_{0}} \sum_{\Delta \mathbf{r}} p(\Delta \mathbf{r})(P(\mathbf{r}-\Delta \mathbf{r}, t)-P(\mathbf{r}, t))
$$

The rate equation can be solved by the standard Fourier transform method [8]:

$$
\begin{aligned}
\frac{\partial \mathscr{F}(P)(\mathbf{q}, t)}{\partial t} & =\frac{1}{\tau_{0}} \sum_{\Delta \mathbf{r}} p(\Delta \mathbf{r})\left(\int \mathrm{d} \mathbf{r}^{\prime} P\left(\mathbf{r}^{\prime}, t\right) \mathrm{e}^{-\mathrm{iq} \cdot\left(\mathbf{r}^{\prime}+\Delta \mathbf{r}\right)}-\int \mathrm{d} \mathbf{r} P(\mathbf{r}, t) \mathrm{e}^{-\mathbf{i q} \cdot \mathbf{r}}\right) \\
& =\mathscr{F}(P)(\mathbf{q}, t) \tau_{0}^{-1} \sum_{\Delta \mathbf{r}} p(\Delta \mathbf{r})\left(\mathrm{e}^{-\mathrm{iq} \cdot \Delta \mathbf{r}}-1\right)
\end{aligned}
$$

To find a solution we need an adequate boundary condition, i.e. the position of a particle of type $B$ at a certain time. The most convenient choice is to let it sit at $\mathbf{r}=\mathbf{0}$ for $t=0$. With this assumption our differential equation yields:

$$
\mathscr{F}(P)(\mathbf{q}, t)=\exp \left(-\frac{1}{\tau_{0}} \sum_{\Delta \mathbf{r}} p(\Delta \mathbf{r})\left(1-\mathrm{e}^{-\mathrm{i} \mathbf{q} \cdot \Delta \mathbf{r}}\right) t\right)
$$

It can now be seen that the Fourier transform $\mathscr{F}(P)(\mathbf{q}, t)$ is nothing more than the intermediate scattering function $g^{(1)}(\mathbf{q}, t)$, see Eq. 6, because the jump probability of an atom in a particular system is the same as the jump probability in an ensemble average. Defining the following term as a correlation time

$$
\tau(\mathbf{q})=\tau_{\text {inc }}(\mathbf{q})=\tau_{0}\left(\sum_{\Delta \mathbf{r}} p(\Delta \mathbf{r})\left(1-\mathrm{e}^{-\mathrm{i} \mathbf{q} \cdot \Delta \mathbf{r}}\right)\right)^{-1},
$$

we can write

$$
g^{(1)}(\mathbf{q}, \Delta t)=\mathscr{F}(P)(\mathbf{q}, \Delta t)=\exp \left(-\frac{\Delta t}{\tau(\mathbf{q})}\right) .
$$

We want to point out that in the simple case above, interactions between the atoms are neglected. Therefore, for this special case, the correlation time $\tau(\mathbf{q})$ is equivalent to the incoherent correlation time $\tau_{\text {inc }}(\mathbf{q})$ measured by quasi-elastic Mössbauer spectroscopy (QEMS) or quasi-elastic neutron scattering (QENS) methods. It has to be stressed that aXPCS is a coherent method and therefore, on general, yields a different correlation time as discussed below. 
Combining Eq. 10 and Eq. 18 we get an equation that allows for relating experimental data used to calculate the intensity autocorrelation function Eq. 7 to a microscopic diffusion model via a $q$ dependent correlation time $\tau(\mathbf{q})$ :

$$
g^{(2)}(\mathbf{q}, \Delta t)=1+\exp \left(\frac{-2 \Delta t}{\tau(\mathbf{q})}\right)
$$

Eq. 19 was deduced under the assumption of perfectly coherent photons, which can never be achieved in a real experiment. To account for this fact a dimensionless coherence factor or Siegert factor, $\beta$, is introduced to Eq. 19 in front of the exponent, see e.g. Ref. [9].

For interpreting experimental results of correlation experiments with coherent photons, Eq. 19 is of central importance. Photon correlation spectroscopy as well as incoherent methods like QENS [2] or QEMS [10] are model-dependent methods. To find an analytical form of the correlation function in Eq. 19, either knowledge of the system or a simple presumption with very few free parameters is needed. Such free parameters are the jump rate of the particle $\tau_{0}^{-1}$ and, if not predetermined by a certain jump model, also the jump probabilities $p(\Delta \mathbf{r})$. The free parameters are adjusted in such a way that the best agreement with the experimentally obtained $g^{(2)}(\mathbf{q}, \Delta t)$ from Eq. 7 is achieved (this is usually done by means of a least squares fit). If the model cannot be matched to the measured data with sufficient precision by adjusting the available free parameters, another model must be considered.

For a system with interacting particles short-range order plays an important role. It should be stressed at this point that XPCS generally and aXPCS in particular are the time Fourier-transformed counterparts of coherent QENS and that concepts developed for QENS can be adopted to XPCS/aXPCS. One only needs to consider that the reciprocal of the correlation time $\tau(\mathbf{q})^{-1}$ corresponds to the line broadening in energy domain $\Gamma(\mathbf{q})$ [2]. In general, the surroundings of each particle influence their movement which means that the inter-particle interactions are not negligible. As a consequence, when using a coherent method like aXPCS, it is necessary to account for the short-range order $I_{\mathrm{SRO}}(\mathbf{q})$. A qualitative argument for including a short-range order dependent term is the so-called de Gennes narrowing [11] which was first introduced for a qualitative description of coherent neutron scattering results in liquids. Physical principles of the de Gennes narrowing are based on the simple observation that peaks in the structure factor of liquids occur at wave vectors corresponding to the most probable interatomic separation. They result also in long-living or, in other words, highly correlated atomic arrangements. The same argument holds for a short-range order in alloys where the scattered radiation has higher intensity in some directions and these intensities correspond to longer lifetimes of certain atomic configurations. A detailed derivation exists only in a linear approximation (i.e. for weak interactions between particles) and can be found in [12] or [13]. Our experimental results to date [14, 15, 16] indicate that this theory works very well and that discrepancies, if any, are negligible with nowadays available coherent beamlines.

The general solution for the correlation time depending on a vector of reciprocal space can be written using $I_{\mathrm{SRO}}(\mathbf{q})$ and $\tau_{\text {inc }}(\mathbf{q})$ from Eq. 17 as

$$
\begin{aligned}
\tau(\mathbf{q}) & =\tau_{0} \frac{I_{\mathrm{SRO}}(\mathbf{q})}{\sum_{n} p_{n} \sum_{\Delta \mathbf{r}_{n j}}\left(1-\exp \left(\mathbf{i q} \cdot \Delta \mathbf{r}_{n j}\right)\right)} \\
& =\tau_{0} \frac{I_{\mathrm{SRO}}(\mathbf{q})}{\sum_{n} \bar{p}_{n}\left(1-Z_{n}^{-1} \sum_{\Delta \mathbf{r}_{n j}} \cos \left(\mathbf{q} \cdot \Delta \mathbf{r}_{n j}\right)\right)},
\end{aligned}
$$

with $Z_{n}$ being the coordination number of a certain neighboring shell and and with $\left\{\Delta \mathbf{r}_{n j}\right\}$ being the family of vectors pointing to the atomic positions in this shell. The probability for the atom to jump to a particular neighboring position is given by $p_{n}$ (the probability to jump to any position within shell $n$ would simply be the product with the number of neighbor atoms in this shell, $\bar{p}_{n}=Z_{n} p_{n}$ ). Note, that in a Bravais lattice for each vector $\Delta \mathbf{r}_{n k}$ there exists a symmetric vector, so that $\Delta \mathbf{r}_{n l}=-\Delta \mathbf{r}_{n k}$ (inversion symmetry), hence the resulting cosine term in Eq. 20. 
Diffusion on a Bravais lattice. In order to calculate Eq. 20 for a specific Bravais lattice we have to consider the family of vectors to neighboring atoms in the $n$th neighbor shell. For cubic lattices this is quite easy and was first obtained by Chudley and Elliott [17]. Let a position of an atom in the lattice be given by $\mathbf{r}=h \mathbf{a}_{1}+k \mathbf{a}_{2}+l \mathbf{a}_{3}$. The family of vectors pointing to the atoms in the $n$th shell is then given by $\{(h, k, l)\}_{n}$. Using $\tau(\mathbf{q})^{-1}=\sum_{n} \tau_{\{(h, k, l)\}_{n}}(\mathbf{q})^{-1}$ we can define $\tau_{\{(h, k, l)\}_{n}}(\mathbf{q})^{-1}$ as:

$$
\tau_{\{(h, k, l)\}_{n}}(\mathbf{q})^{-1}=\frac{\bar{p}_{n}}{\tau_{0} I_{\mathrm{SRO}}(\mathbf{q})}\left(1-\frac{1}{Z_{n}} \sum_{\Delta \mathbf{r}_{n}} \cos \left(\mathbf{q} \cdot \Delta \mathbf{r}_{n}\right)\right)
$$

The calculation of the sum in Cartesian coordinates $\left(\mathbf{q}=q_{x} \hat{x}+q_{y} \hat{y}+q_{z} \hat{z}\right)$ can be considerably simplified:

$$
\begin{aligned}
& \tau_{\{(h, k, l)\}_{n}}(\mathbf{q})^{-1}=\frac{\bar{p}_{n}}{\tau_{0} I_{\mathrm{SRO}}(\mathbf{q})} \quad\left(1-\frac{1}{6}\left(\cos \left(q_{x} h\right) \cos \left(q_{y} k\right) \cos \left(q_{z} l\right)+\cos \left(q_{x} h\right) \cos \left(q_{y} l\right) \cos \left(q_{z} k\right)\right.\right. \\
& +\cos \left(q_{x} k\right) \cos \left(q_{y} h\right) \cos \left(q_{z} l\right)+\cos \left(q_{x} k\right) \cos \left(q_{y} l\right) \cos \left(q_{z} h\right) \\
& \left.\left.+\cos \left(q_{x} l\right) \cos \left(q_{y} h\right) \cos \left(q_{z} k\right)+\cos \left(q_{x} l\right) \cos \left(q_{y} k\right) \cos \left(q_{z} h\right)\right)\right)
\end{aligned}
$$

As an example, consider nearest-neighbor jumps in an fcc and in a bcc lattice,

$$
\begin{aligned}
\tau_{\left\{\left(\frac{a}{2}, \frac{a}{2}, 0\right)\right\}_{1}}^{f c c}(\mathbf{q})^{-1}=1- & \frac{1}{3}\left(\cos \left(\frac{1}{2} a q_{x}\right) \cos \left(\frac{1}{2} a q_{y}\right)+\cos \left(\frac{1}{2} a q_{x}\right) \cos \left(\frac{1}{2} a q_{z}\right)\right. \\
& \left.+\cos \left(\frac{1}{2} a q_{y}\right) \cos \left(\frac{1}{2} a q_{z}\right)\right), \\
\tau_{\left\{\left(\frac{a}{2}, \frac{a}{2}, \frac{a}{2}\right)\right\}_{1}}^{b c c}(\mathbf{q})^{-1}= & 1-\cos \left(\frac{1}{2} a q_{x}\right) \cos \left(\frac{1}{2} a q_{y}\right) \cos \left(\frac{1}{2} a q_{z}\right),
\end{aligned}
$$

where $a$ is the lattice constant. In Fig. 1 the inverse correlation times for fcc and bcc nearest-neighbor jumps are plotted on an Ewald sphere and as a spherical projection for particular experimental conditions.

In a solid solution with a very low concentration of foreign atoms aXPCS, following the movement of the solute atoms, measures a diffusivity corresponding to the self-diffusion coefficient. If the concentration of the foreign atoms becomes higher, aXPCS measures chemical fluctuations. The measured diffusivity therefore corresponds to the so-called chemical diffusion coefficient. More details in Section Self-diffusion and Section Chemical diffusion.

Diffusion on a non Bravais lattice. A thorough discussion of the nontrivial case of diffusion on lattices with nonequivalent lattice sites is beyond the scope of this review. Thus we only present a brief summary with an extensive reference list. In a number of alloys with non-negligible interactions between constituents lattice sites are not occupied randomly but exhibit an additional type of order in the form of a superlattice. Two or more sublattices are occupied by different elements and the degree of order is controlled by the stoichiometry and temperature. These so-called intermetallic alloys like Ti-Al, Fe-Al, Ni-Al, Ni-Fe-Al and many others are of high technological interest [18]. In intermetallic alloys, jumps of a given atomic species onto another sublattice may be less probable than jumps to sites on the own sublattice, which can lead to multiple jump rates. Therefore diffusion mechanisms in intermetallic alloys are usually more complicated than in solid solutions. Even if all lattice sites are energetically equivalent but crystallographically different (like for instance in the silica lattice) it is not sufficient to set up only one differential equation in the form of Eq. 15 to find the intermediate scattering function. For a superstructure with $m$ sublattices one needs $m$ rate equations which can be solved applying a matrix notation using an $m \times m$ matrix $\tilde{\mathbf{A}}$ :

$$
\frac{\partial}{\partial t} \mathscr{F}(P)(\mathbf{q}, t)=\tilde{\mathbf{A}} \mathscr{F}(P)(\mathbf{q}, t)
$$



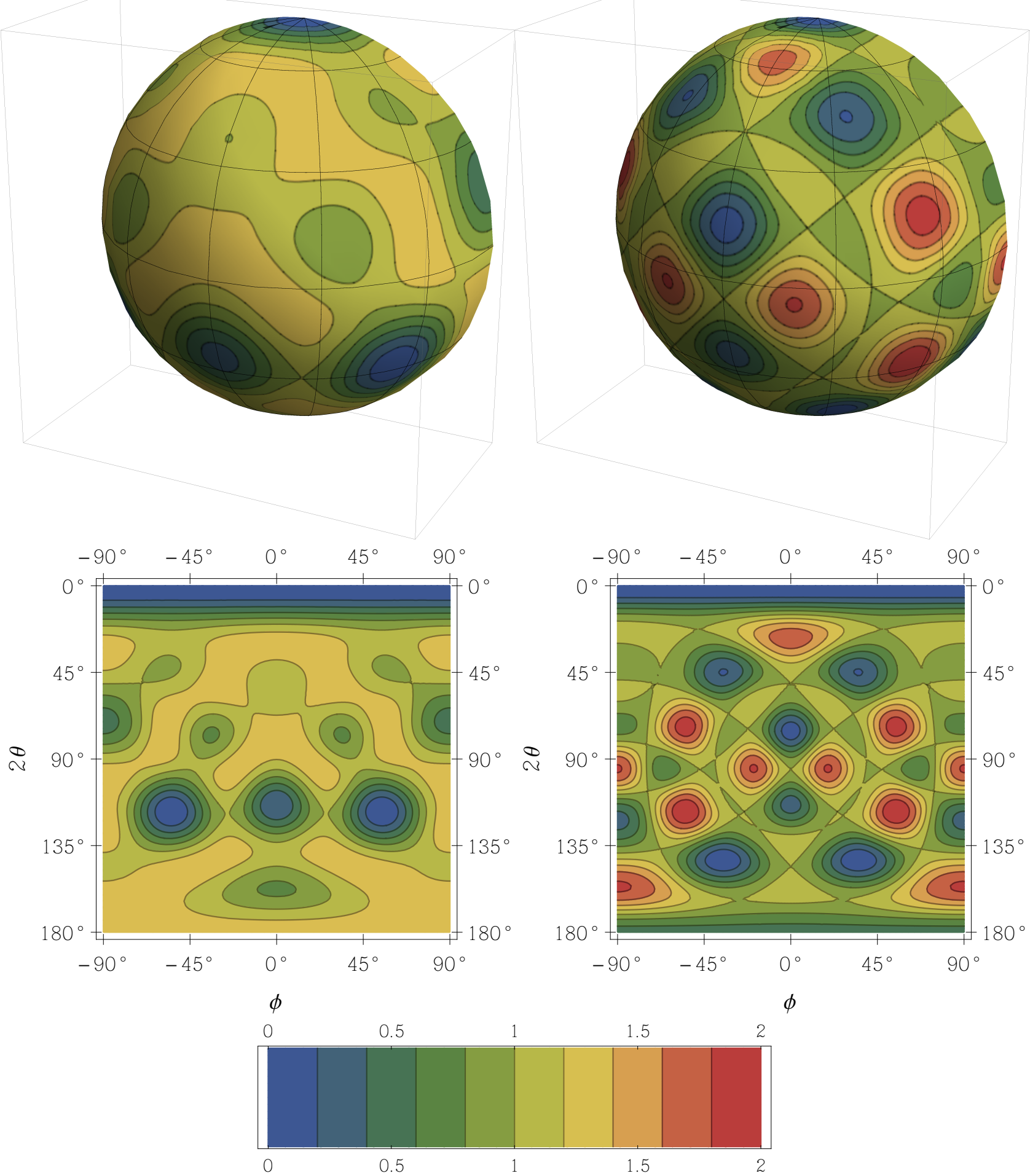

Fig. 1: Contour plots of inverse correlation times $\tau^{-1}(\mathbf{q})$ in arbitrary units, calculated for an $8 \mathrm{keV}$ X-ray beam $\left(\mathbf{k}_{\mathrm{in}}\right)$ parallel to the $\langle 110\rangle$ direction of a sample with a lattice constant of $a=3 \AA$. Left: Modeled fcc nearest neighbor jumps $\left(\frac{a}{2}, \frac{a}{2}, 0\right)$. Right: Modeled bcc nearest neighbor jumps $\left(\frac{a}{2}, \frac{a}{2}, \frac{a}{2}\right)$. The Ewald spheres and their spherical projections are shown. 
The matrix $\tilde{\mathbf{A}}$ describing the dynamics in non-Bravais lattice is called jump matrix and is defined as

$$
\tilde{\mathbf{A}}=\left(Z_{j i} \tau_{j i}\right)^{-1} \sum_{k} \exp \left(-\mathbf{i q} \Delta \mathbf{r}_{i j k}\right)-\delta_{i j} \sum_{j}\left(\tau_{i j}\right)^{-1}
$$

where the vector $\Delta \mathbf{r}_{i j k}$ connects the site of local symmetry $i$ and the $k$ th site of local symmetry $j$. $Z_{i j}$ is the number of the $j$ th sublattice sites surrounding the $i$ th sublattice and $\tau_{i j}^{-1}$ the jump rate from a site of symmetry $i$ to any nearest-neighbor site of symmetry $j$. The jump matrix is Hermitian only if $Z_{i j} \tau_{i j}=Z_{j i} \tau_{j i}$ but it can be Hermitized by a similarity transformation. Moreover, it has always real eigenvalues [19].

The problem of a theoretical description of diffusion in non-Bravais lattices has been solved in a number of papers [20, 19, 21] and is treated in depth by Randl et al. [22] and in Sepiol and Ludwig [23]. In a nutshell, the intermediate scattering function for $m$ sublattices is a sum of $m$ exponential functions (i.e., it is a non-exponential function) with time constants $M_{p}$ being the eigenvalues of the matrix $\tilde{\mathbf{A}}$

$$
\mathscr{F}(P)(\mathbf{q}, t)=\sum_{p} w_{p} \exp \left(M_{p} t\right), w_{p}=\left|\sum_{i} \sqrt{c_{i}} b_{p}^{i}\right|^{2},
$$

and weighting factors $w_{p}$ calculated from the components $b_{p}^{i}$ of the $p$ th eigenvector of the jump matrix [22]. The quantity $c_{i}$ is the occupation of the sublattice $i$, therefore $\sum_{i} c_{i}=1$.

One should note that direct experimental observation of a multi-exponential decay is a difficult task and more the exception than the rule. Using quasi-elastic methods one measures in the energy domain instead of in the time domain, thus a sum of exponents is equal to a sum of Lorentzian functions. Because these two domains are connected via a Fourier transform, the theory is the same. Measurements of signals composed of more than one Lorentzian were achieved with Mössbauer spectroscopy and with nuclear resonant scattering methods in $\mathrm{Fe}_{3} \mathrm{Si}$ and $\mathrm{Fe}-\mathrm{Al}$ [24, 25, 26], with QENS in Ni-Ga and in Co-Ga intermetallic alloys $[27,28]$ and directly in the time domain with neutron spin-echo spectroscopy in Ni-Ga [29, 30].

Diffusion in amorphous materials. Contrary to a single crystal, amorphous or polycrystalline samples are isotropic and have no inner symmetry on large scales. This isotropy can be seen as an average over all possible alignments of an infinite number of locally oriented crystals. Instead of performing an average over all possible orientations in real space, one can average over all possible directions of the incoming beam vector $\mathbf{k}_{\text {in }}$. This is equivalent to an average over all possible orientations of the scattering vector: $\left|\mathbf{k}_{\text {out }}-\mathbf{k}_{\text {in }}\right| \equiv|\mathbf{q}|=\Lambda^{-1} \int_{0}^{4 \pi} \mathrm{d} \Omega \mathbf{q}$. Applying this to Eq. 19 yields a correlation function that is only dependent on the absolute value of the scattering vector $|\mathbf{q}|$, which in turn depends on the scattering angle $2 \theta$. It would be worthwhile to perform this calculation straightforwardly,

$$
\tilde{g}_{\text {ana }}^{(2)}(\mathbf{q}, \Delta t)=1+C_{\text {norm }} \int_{|\mathbf{q}|=q} \mathrm{dq} \exp \left(\frac{-2 \Delta t}{\tau(\mathbf{q})}\right),
$$

but unfortunately no analytic solution can be found for Eq. 28. Therefore an approximation has to be made, where the correlation time is averaged over all possible directions of the q-vector yielding $\tilde{\tau}(q)$, rather than averaging over the exponential function:

$$
\tilde{g}^{(2)}(\mathbf{q}, \Delta t):=1+\exp \left(\frac{-2 \Delta t}{\tilde{\tau}(q)}\right) \approx \tilde{g}_{\mathrm{ana}}^{(2)}(\mathbf{q}, \Delta t)
$$

Finding $\tilde{\tau}(q)$ is not straight forward, because there are two $q$-dependent terms in $\tau(\mathbf{q})$ (Eq. 20) which cannot be analytically separated in a general way. Therefore yet another simplification has to be applied:

$$
C_{\text {norm }}^{\prime} \int_{|\mathbf{q}|=q} \operatorname{dq} \tau(\mathbf{q}) \approx \tau_{0} \frac{C_{\text {norm }}^{\prime \prime} \int_{|\mathbf{q}|=q} \mathrm{~d} \mathbf{q} I_{\text {SRO }}(\mathbf{q})}{C_{\text {norm }}^{\prime \prime \prime} \int_{|\mathbf{q}|=q} \operatorname{dq} \sum_{n} p_{n} \sum_{\Delta \mathbf{r}_{n j}}\left(1-\exp \left(\mathbf{q} \cdot \Delta \mathbf{r}_{n j}\right)\right)}=: \tilde{\tau}(q)
$$



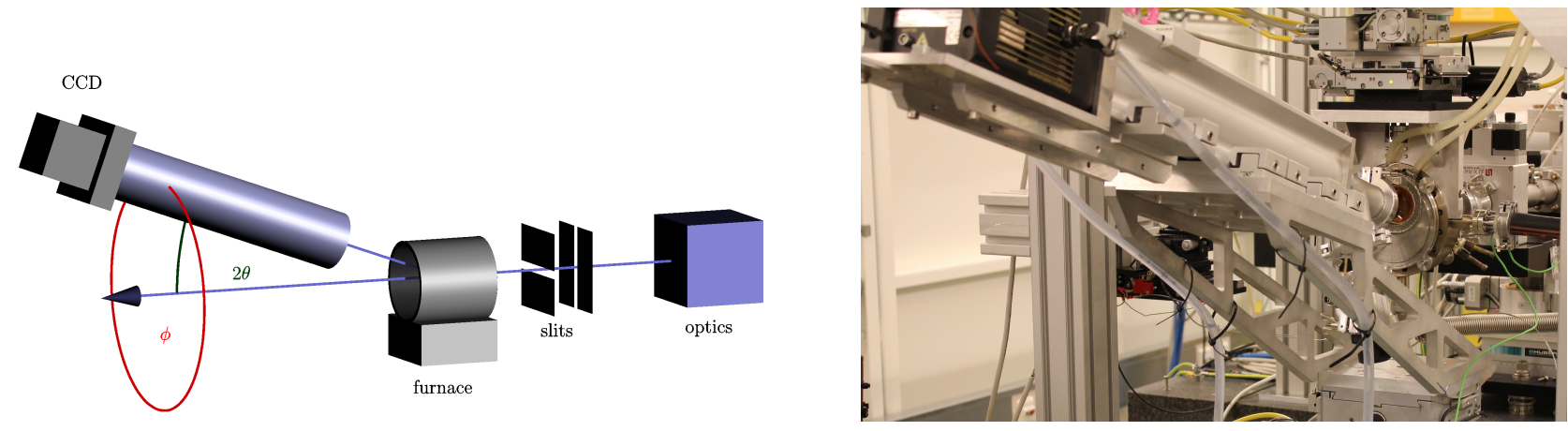

Fig. 2: Left: Schematic representation of the aXPCS experimental setup. The free parameters in the experiment are the scattering angle $2 \theta$, the azimuthal angle $\phi$ of the CCD camera versus the beam, the sample to detector distance $d$, the exposure time and the sample temperature. Right: Picture of the actual setup at PETRA III, Hamburg.

Here the numerator gives the average intensity $\tilde{I}_{\mathrm{SRO}}(q)$. The integral in the denominator can be explicitly written as $(4 \pi)^{-1} \int_{0}^{2 \pi} \mathrm{d} \phi \int_{0}^{\pi} \mathrm{d} \theta(1-\exp (\mathrm{i} q \Delta r \cos (\theta)))=1-\frac{\sin (q \Delta r)}{q \Delta r}$. This result is very similar to the well known Chudley-Elliott model form [17]. The only alteration is the inclusion of the average short-range order intensity in the denominator:

$$
\tilde{\tau}(q)=\tau_{0} \frac{\tilde{I}_{\mathrm{SRO}}(q)}{\sum_{n} p_{n} \sum_{\Delta \mathbf{r}_{n j}}\left(1-\frac{\sin \left(q \Delta r_{n j}\right)}{q \Delta r_{n j}}\right)}=\tau_{0} \frac{\tilde{I}_{\mathrm{SRO}}(q)}{\sum_{n} \bar{p}_{n}\left(1-\frac{\sin \left(q \Delta r_{n j}\right)}{q \Delta r_{n j}}\right)}
$$

\section{Experimental setup}

In the experiment a coherent X-ray beam is scattered by a sample and the scattering intensity is collected by a CCD camera as shown in Fig. 2. An X-ray beam smaller than $10 \times 10 \mu \mathrm{m}^{2}$ is used. This can be achieved by using rollerblade slits before the sample or, if possible, by focusing the beam [31]. At a given wavelength the dominating factors for the size of the projected speckles are the beam size and the distance from the sample. The sample to detector distance is optimized according to the pixel size of the CCD. Even though the coherence factor increases if the speckles cover more than one pixel, it is desirable to have the speckle size matching the pixel size in order to increase the count rate (for more details see Leitner [32]). This is especially important for weakly scattering samples as is commonly the case in intermetallic phases. To maximize intensity, the sample thickness should ideally match the scattering length. However, a sample thickness much larger than the longitudinal coherence length of the beam drastically reduces the coherence factor. In this case, contrary to above's case of optimizing the sample to detector distance, coherence is usually favored over intensity by making the samples thinner. This is best achieved by using a dimpling grinder as it is common in the sample preparation for transmission electron microscopy experiments. The temperature of the sample is controlled in a furnace or in a cryostat and should be stabilized around $0.1 \mathrm{~K}$.

Even with best of today's X-ray sources, coherent intensity is still rather low. Only single photon events are therefore recordable under large scattering angles in the time scales of our experiments. In order to improve statistics, the use of an area detector is indispensable. A droplet algorithm [33] is used to identify the photon events and all points lying within the area of the detector are treated as equivalent in the reciprocal space and all are used to calculate an ensemble average. This is reasonable because for high X-ray energies the Ewald sphere is flat for the solid angle occupied by the area detector and also gradients of the inverse correlation time in the diffusion models are rather flat as shown in Fig. 1.

Note that in principle there is no limitation in terms of elements accessible for aXPCS. With today's synchrotron sources, however, one of the scattering lengths needs to be significantly different from the other(s) in order get sufficient statistics. There is also no primal limitation to timescales accessible with 
aXPCS. Towards slow dynamics the timescale is simply limited by the stability of the experimental setup and the time the sample stays in an equilibrium state. The main limiting factor for aXPCS towards fast dynamics is the intensity of the beam, but what limits time resolution even more with current setups is the readout time of the CCD detector. Although there are ways to partly circumvent this, like e.g. using the kinetic mode of a camera [34], the introduction of new CCDs with considerately shorter readout times like the EIGER detector [35] promises to be a revolutionary improvement.

The exact experimental approach would go beyond the scope of this review. However, the following criteria have to be met in order for an experiment to work without addenda to the above theory section. Firstly, the atomic jumps have to occur on timescales negligible in comparison to residence times. Secondly, thermal diffuse scattering has to be low in comparison to the short-range order intensity. Moreover, correlation of the Warren-size-phenomena has to be zero. Last but not least incoherent scattering effects should be negligible and the scattering should be purely elastic.

\section{Self-diffusion}

From a theoretical point of view the simplest kind of diffusion is a self-diffusion, i.e. a motion of atoms on their own lattice [36] in a chemically homogeneous material. This kind of diffusion can, in practice, not be measured and special tagged atoms, usually atomic isotopes other than the matrix atoms, are used to trace their motion and to find out diffusion coefficients and/or the diffusion mechanism. Today there is no doubt that the vehicle of self-diffusion in metals are vacancies $[36,37]$. The model to describe self-diffusion of an atom in a matrix via a vacancy was introduced by Wolf [38] and is called the encounter model.

$\mathbf{A ~ N i} \mathbf{~ i}_{0.97} \mathbf{P t}_{0.03}$ solid solution. Diffusion in a solid solution with concentrations of only few percent of dilute atoms can be very similar to self-diffusion. This is the case if there is negligible interaction between the dilute atoms and the matrix atoms and if the concentration is low enough so that encounters of the dilute atoms are very unlikely. In such a case the encounter model provides a good estimation for jump probabilities. This was shown for a $\mathrm{Ni}_{0.97} \mathrm{Pt}_{0.03}$ solid solution, where the dominating interaction is a repulsive force between the Pt atoms, as shown in Stana et al. [15].

A computer aXPCS experiment was carried out to show the influence of short-range order even in dilute systems with relatively low concentrations of foreign atoms. An fcc lattice with $4 \times 32^{3}$ atoms with periodic boundary conditions was randomly filled with one vacancy and $\mathrm{Ni}$ and $\mathrm{Pt}$ atoms in a ratio 97:3. The interaction between $\mathrm{Ni}-\mathrm{Ni}$ and $\mathrm{Ni}-\mathrm{Pt}$ was neglected while the parameters for the $\mathrm{Pt}-\mathrm{Pt}$ interaction were controlled.

A conventional Metropolis Monte Carlo algorithm (see, e.g. Haider et al. [39]) was applied to calculate jump probabilities between atoms. After annealing the system, short-range order parameters were calculated. Then after every tenth of a Monte Carlo step a Fourier transform of the system was carried out and the so-computed amplitudes were used to calculate the intensities for a certain point in reciprocal space.

These intensities were used to calculate the intensity autocorrelation function according to Eq. 7 for several points in reciprocal space. This was done for no interaction between Pt atoms, for strong repulsion and for an attractive force, respectively. Note that the attractive force was a factor of 10 smaller than the repulsive force in order to avoid fast clustering. The results were compared with the encounter model corrected by the short-range order intensity. This intensity (in Laue units) was calculated from Warren-Cowley SRO parameters $\alpha_{i}$ [40] computed in the simulation, using

$$
I_{\mathrm{SRO}}(\mathbf{q})=1+\sum_{n, j} \alpha_{n} \cos \left(\mathbf{q} \cdot \Delta \mathbf{r}_{n j}\right)
$$

where $\Delta \mathbf{r}_{n j}$ is the family of vectors pointing to positions in the $n$ th-neighbor shell (starting with $n=1$ for nearest neighbors). Note that for the case of an attractive force between dilute atoms no short-range order parameters were calculated, therefore only the data from the computer experiment are given in 

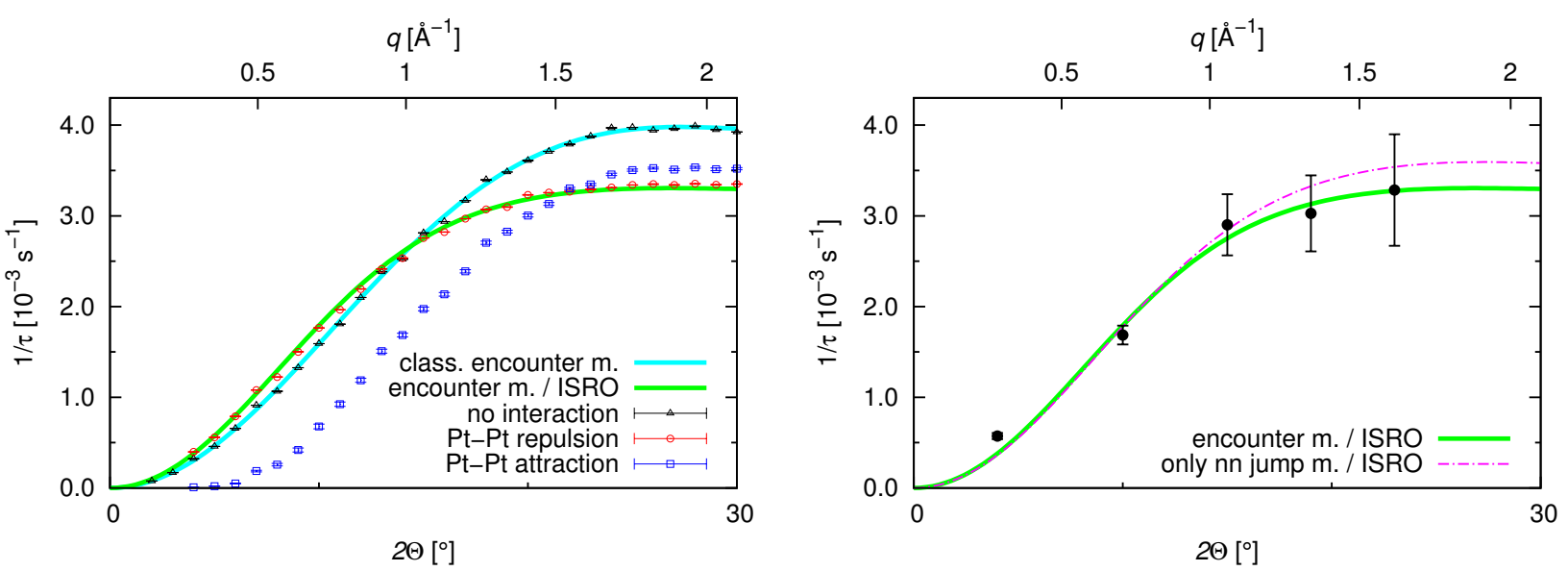

Fig. 3: Left: The encounter model as introduced by Wolf was calculated analytically using renormalized jump probabilities $\left(\bar{p}_{1}=0.926, \bar{p}_{2}=0.24, \bar{p}_{3}=0.39\right.$ and $\left.\bar{p}_{4}=0.08\right)$ and corrected for short-range order by means of Warren-Cowley SRO parameters. These parameters were computed from Monte Carlo simulations. The models were compared with data obtained from computer experiments on the basis of aXPCS experiments. Right: The encounter model and a model with only nearest-neighbor jumps $\left(\bar{p}_{1}=1\right)$, both corrected for short-range order in a system with strong Pt-Pt repulsion, compared with experimental data as published in [15].

Fig. 3. From the other two cases it can be seen however, that the computer aXPCS experiment and the analytical encounter model corrected by SRO match very well.

In the right part of Fig. 3 the encounter model corrected for short-range order for a repulsive force is compared with experimental results as published in [15]. Also a model with only nearest neighbor jumps and corrected for SRO in the same way is shown. The experiment was not able to distinguish between both models. However with improving synchrotron sources and shrinking measurement errors this will be possible in the future. Other models like sole next-nearest neighbor jumps can, however, definitely be disqualified, as discussed in the original paper.

As demonstrated in [15] aXPCS experiments can also be carried out using polycrystalline samples. However, there are drawbacks which are be discussed briefly. From a theoretical point of view single crystalline samples yield more information, because additional to a rocking-curve-scan an azimuthal scan can be carried out. From an experimental point of view the number of crystallites within the volume illuminated by the X-ray beam has to be sufficiently high in order to allow for averaging over all crystallographic directions. When dealing with solid solutions it is difficult to produce bulk samples with this quality. It is even more difficult to prevent the growth of the crystallites during the experiment. Therefore another approach will be followed in the future. The material will be ground to a fine powder to gain a large number of small $\mu \mathrm{m}$ size crystals as it is done for powder diffraction. This could proof very useful for samples where growing single crystals is very difficult or even impossible.

\section{Chemical diffusion}

The description of systems which are chemically inhomogeneous is much more complicated. From a technological point of view, however, these are the systems where gaining knowledge about diffusion is especially important. Generally speaking the fluxes of different species in such systems are not independent but are coupled so that an effective diffusion coefficient describing mutual influence of two or more components has to be introduced [36]. There can be different reasons for such a coupling, e.g. the fact that the homogeneous composition has to be conserved also on the microscopic scale. Apart from coupling, another reason for introducing an effective diffusion coefficient is that the motion of one component of the alloy cannot be treated separately from the motion of another component. 
Regarding the nomenclature of such a process one must be careful because different names are commonly used. Owing to the most frequently applied experimental method for diffusion studies, which is the tracer method, interdiffusion is the most widely used denomination. The type of tracer method this name is owed to is based on the following principle: a diffusion couple of two samples, e.g. $A_{c} B_{1-c}$ alloys of different stoichiometry, is made and annealed at distinct temperatures. The resulting concentration profiles of $\mathrm{A}$ and $\mathrm{B}$ components interdiffusing through the intersection plane are analyzed providing the concentration-dependent interdiffusion coefficient $\tilde{D}(x)$. Because of the temperature as well as the concentration dependence of the process, a very detailed two-dimensional knowledge of the diffusion coefficient can be obtained which additionally includes activation enthalpies of $\mathrm{A}$ and $\mathrm{B}$ species.

Another, probably more intuitive name for $\tilde{D}$ is chemical diffusion coefficient. It is owed to the fact that global concentration gradients of $\mathrm{A}$ and $\mathrm{B}$ components are not a prerequisite for atomistic or macroscopic diffusion but that even in a macroscopically homogeneous system diffusion takes place. The composition gradient is only a practical requirement for diffusion to be observable [41] but not an essential condition for it at all. The term collective diffusion [2] appears even more suitable because it does not suffer from this misunderstanding. It is, however, not very widespread in the literature. We will therefore use the name chemical diffusion from here on and limit it to binary systems only.

Coherent methods of diffusion studies like aXPCS or coherent QENS measure a chemical diffusion coefficient $\tilde{D}$, contrary to the incoherent methods like QEMS, incoherent QENS or spin-echo neutron spectroscopy which measure self-diffusion of selected isotopes like ${ }^{57} \mathrm{Fe},{ }^{119} \mathrm{Sn}$ or of few exclusive QENS elements scattering predominantly incoherently, like $\mathrm{H}$, Ti, or Ni. The self-diffusion coefficient $D_{\mathrm{s}}$ connects macroscopical and atomistic diffusion via the famous Einstein-Smoluchowski relation

$$
D_{s}=\frac{|\Delta \mathbf{r}|^{2}}{6 \tau_{0}},
$$

where $|\Delta \mathbf{r}|$ the jump length and $\tau_{0}^{-1}$ the jump rate. In contrast, chemical diffusion can not be interpreted in a straightforward way. A useful equation connecting the chemical diffusion $\tilde{D}$ in a binary system with the two tracer diffusion coefficients $D_{\mathrm{A}^{*}}$ and $D_{\mathrm{B}^{*}}$ in an alloy was derived by Darken [42]

$$
\tilde{D}=\left(c_{\mathrm{B}} D_{\mathrm{A}^{*}}+c_{\mathrm{A}} D_{\mathrm{B}^{*}}\right) \varphi S_{\mathrm{v}},
$$

where $\varphi$ is the thermodynamic factor involving gradients of chemical potential $\mu$ of substituents $(\varphi=$ $\left.1+\partial \ln \mu_{\mathrm{A}} / \partial \ln c_{\mathrm{A}}\right)$ and $S_{\mathrm{v}}$, which is called the vacancy wind, describes the subtle correlation between movements of constituent atoms and vacancies. Manning [43] analyzed the last factor for a binary random alloy and for isolated vacancies deriving an analytical expression for $S_{\mathrm{v}}$. The Darken relation and the Manning relation have been widely applied to link experimental diffusion in alloys to its microscopic picture. Most of the theoretical works have centered around $\mathrm{B} 1(\mathrm{NaCl})$ and $\mathrm{B} 2(\mathrm{CsCl})$ structures with only intersublattice jumps, see e.g. Murch and Belova [41]. In this review however we concentrate on Ni-rich Ni-Pt alloys, which show a tendency to create the $\mathrm{L} 1_{2}$-type structure on the short-range scale. Most of the work in the $\mathrm{L}_{2}$ structure has been done by Belova and Murch [44] using computer simulations to calculate the $S_{\mathrm{v}}$-factor in three compositions close to the $3: 1$ stoichiometry and in a wide range of vacancy-exchange frequencies between A and B atoms. The Manning formalism was confirmed in these simulations [44] to generally work very well.

The Ni-rich side of $\mathbf{N i}_{1-c} \mathbf{P t} \mathbf{t}_{c}$ intermetallic alloys. In this review we conduct a computer simulation of an aXPCs experiment similar to the method developed by Belova and Murch [41, 44] to calculate the chemical diffusion coefficient $\tilde{D}$

$$
\tilde{D}=\frac{\left\langle\left(c_{\mathrm{B}} \Delta \mathbf{R}_{\mathrm{A}}-c_{\mathrm{A}} \Delta \mathbf{R}_{\mathrm{B}}\right)^{2}\right\rangle \varphi}{6 t N c_{\mathrm{A}} c_{\mathrm{B}}},
$$

where $\Delta \mathbf{R}_{i}$ is the sum of the displacements of all atoms of type $i$ during time $t$ and $N$ is the number of particles. The vacancy-wind factor calculated in the $\mathrm{L}_{2}$-type structures [44] was very well consistent 
with the Darken/Manning relation Eq. 34 and differed only slightly for very large, unreasonable ratios between jump frequencies of the components. On the other hand the thermodynamic factor $\varphi$ is small in weakly interacting alloys as claimed in [36]. Consequently we will neglect these factors in the following consideration.

A Monte Carlo simulation was carried out to gain information about the degree of order of $\mathrm{L}_{2}$-type alloys and about the atomic mobility in the systems. The sample was simulated by arranging nickel and platinum atoms in proportions of 3,10 and 20 at.\% platinum over an fcc-type lattice containing $4 \times 32^{3}$ sites with periodic boundary conditions and with one vacancy. The distance between nearestneighbor atoms of the fcc structure was $\sqrt{2}$ in dimensional units. Atoms were allowed to diffuse on the lattice by use of a Metropolis algorithm as discussed in Section Self-diffusion.

Although the Ni-Pt system nominally shows three ordered equilibrium phases in the phase diagram, namely $\mathrm{NiPt}\left(\mathrm{L}_{0}\right.$ structure), $\mathrm{Ni}_{3} \mathrm{Pt}$, and $\mathrm{NiPt}_{3}$ (both $\mathrm{L}_{2}$ structures) [45], only a very weak tendency could be found for a $\mathrm{Cu}_{3} \mathrm{Au}$-type ordering for $\mathrm{Ni}_{1-c} \mathrm{Pt}_{c}$ with $c=0.2$ composition [46]. Unfortunately there exists no reliable data for SRO parameters on a system with these stoichiometries in the literature. To our knowledge, only measurements for Pt concentrations of 20 and 30 at.\% [47] were performed on polycrystalline samples which were evaluated with a method based on the rather outdated approach introduced by Warren [48]. Based on actual small-angle measurements that we conducted on $\mathrm{Ni}_{0.97} \mathrm{Pt}_{0.03}$ samples [15] and on preliminary ab initio calculations [49] it is known that the repulsive force between Pt nearest-neighbor atoms is quite sufficient to describe SRO in this structure. Moreover, for this system calculating the Warren-Cowley SRO parameters directly from the simulation data without taking care of the local displacements in the lattice yields reasonable results. In general the analysis of diffuse scattering is laborious and time consuming due to the challenging task of separating SRO scattering from other, higher order intensity-terms like the atomic displacement scattering, Huang scattering or thermal diffuse scattering. In order to keep the model close to the reality but still as simple as possible, we choose only one ordering parameter $J^{(1)}=\frac{1}{4}\left(\epsilon_{\mathrm{AA}}^{(1)}+\epsilon_{\mathrm{BB}}^{(1)}-2 \epsilon_{\mathrm{AB}}^{(1)}\right)=1$, where $\epsilon_{X Y}^{(n)}$ are pair interactions between $\mathrm{A}$ and B atoms $(X, Y \in\{\mathrm{A}, \mathrm{B}\})$ up to the $n$th neighbor distance (see Weinkamer et al. [50] for more details). The temperature was set to $T=2 J^{(1)}$ and we ensured that this temperature was higher than the critical temperature of ordering for all three concentrations of 3, 10 and 20 at.\% (for instance in $c=20$ at.\% alloy the critical temperature was 1.47 ).

The first step in the simulation was equilibrating the system and selecting a number of WarrenCowley SRO parameters $\alpha_{i}$ from the atomic configurations. These parameters were used to calculate the short-range order intensity according to Eq. 32. The number of order parameters $\alpha_{i}$ was limited to 13, 4 and 3 for 20, 10 and 3 at.\% Pt alloys, respectively. This was sufficient, because for higher numbers the values of $\alpha_{i}$, decreasing with increasing $i$, are smaller than $10^{-2}$. Therefore they do not contribute significantly to the scattering intensity and can safely be neglected.

After equilibrating the system, the actual diffusion simulation was started. After each Monte Carlo step (or a fraction of an MC step) a fast Fourier transform of the lattice was carried out and the scattering amplitudes gained thereby were used to calculate the simulated scattering intensities. These intensities were used to calculate the autocorrelation function $g^{(2)}(\mathbf{q}, \mathbf{t})$ using Eq. 7 for a certain $\mathbf{q}$ vector exactly as it is done in an actual aXPCS experiment. The q vectors were selected along a circle with a radius of $2 \pi / a$ in the (110) crystal plane. The resulting autocorrelation functions were fitted according to Eq. 19, providing correlation times $\tau_{\mathrm{MC}}(\mathbf{q})$. The reciprocal correlation times $\tau_{\mathrm{MC}}(\mathbf{q})^{-1}$ which are equivalent to the line broadening $\Gamma(\mathbf{q})$ in energy domain are consequently fitted with the general solution for diffusion in interacting systems, Eq. 20. There are just two sets of fit parameters: the atomic jump frequency $\tau_{0}^{-1}$ and the probabilities for the atom to jump to a particular neighboring shell $\bar{p}_{n}$. All other factors in Eq. 20 are of purely geometrical nature. Note that for a particular model, e.g. the encounter model as discussed in Section Self-diffusion also the jump probabilities are predetermined, leaving only one fit parameter $\tau_{0}$. The results of the simulations are presented in Fig. 5 . 


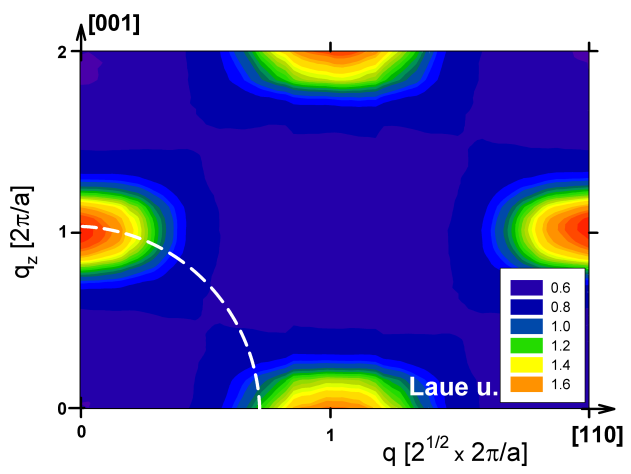

Fig. 4: Cut through the (110) crystal plane showing $I_{\text {SRO }}$ intensity calculated for a 20 at.\% Pt fcc solid solution with repulsive force between neighboring platinum atoms. Intensities are shown in Laue units and the broken line highlights the q-values for which the autocorrelation values were simulated.

In parallel the SRO intensity was obtained as shown in Fig. 4. Diffuse-intensity peaks emerging at q-values corresponding to the $\mathrm{L}_{2}$ superstructure Bragg positions are clearly visible (fcc Bragg peaks were removed for better visibility). Fig. 5 stresses the importance of the SRO in the interpretation

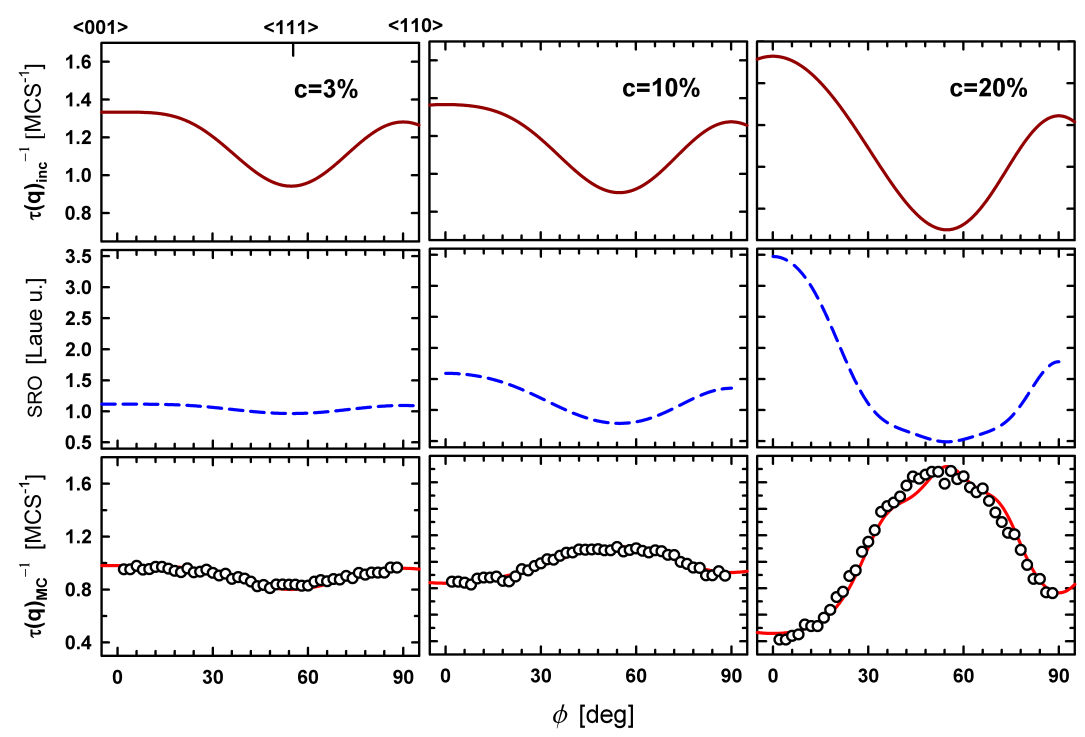

Fig. 5: Three different $\mathrm{Ni}_{1-c} \mathrm{Pt}_{c}$ alloys were simulated (left to right). The upper row (continuous, dark red line) shows the inverse incoherent correlation time $\tau_{\text {inc }}(\mathbf{q})^{-1}$ calculated from Eq. 17, the middle row (broken, blue line) shows $I_{\mathrm{SRO}}(\mathbf{q})$ calculated from Eq. 32. The lower figures show Monte Carlo simulated $\tau_{\mathrm{MC}}^{-1}(\mathbf{q})$, fitted with $\frac{\tau_{\text {inc }}(\mathbf{q})^{-1}}{I_{\mathrm{SRO}}(\mathbf{q})}$. The simulations were performed as a function of the azimuthal angle $\phi$ in the (110) plane (see Fig. 4). Here $\phi=0^{\circ}$ corresponds to the q-vector along the $\langle 001\rangle$ direction, $\phi \cong 55^{\circ}$ to the $\langle 111\rangle$ and $\phi=90^{\circ}$ to the $\langle 110\rangle$ crystal direction.

of correlation times resulting from aXPCS measurements. In the presented Monte Carlo simulation we know that the underlying atomistic diffusion mechanism is a simple nearest-neighbor exchange with a vacancy. The only variable which discriminates between the left, middle and right column in Fig. 5 is the alloy stoichiometry (note that temperature and pair-interaction parameters are kept the same). The diffuse intensity $I_{\mathrm{SRO}}(\mathbf{q})$ which is more and more pronounced with the growing $\mathrm{Pt}$ content, reversed the curvature of the $\tau_{\mathrm{MC}}^{-1}(\mathbf{q})$, whereas the $\tau_{\text {inc }}^{-1}(\mathbf{q})$ which is directly reflecting the atomistic jump mechanism, stays virtually unchanged. 
Special attention should be paid to a particular feature of the fitting procedure. As mentioned above, the atomic jump frequency $\tau_{0}^{-1}$ was not the only fit parameter but also the atomic jump probabilities $\bar{p}_{n}$ were fitted. The results for 3 at.\% Pt (left column of Fig. 5) could be fitted with a pure nearestneighbor jump model, $\bar{p}_{1}=1, \bar{p}_{n \neq 1}=0$. The fit for the 10 at.\% Pt alloy was improved with a small $5 \%$ admixture of next-nearest-neighbor jumps, i.e. jumps along the edge of the cubic unit cell $(\langle 100\rangle$ direction). The fit of 20 at. $\%$ Pt was possible only with about $20 \%$ admixture of $\langle 100\rangle$ next-nearestneighbor jumps, but of no further jumps $\left(\bar{p}_{1}=0.8, \bar{p}_{2}=0.2\right)$. The interpretation is as follows: platinum atoms in a 3 at.\% Pt Ni-Pt alloy are sufficiently diluted so that the interactions between soluble atoms are very weak (see Fig. 5 left column with a flat $I_{\mathrm{SRO}}(\mathbf{q})$ line). Nearest-neighbor jumps describe atomistic diffusion satisfactorily. Increasing the Pt content leads to stronger SRO-interactions. The repulsive force between Pt atoms determines that platinum nearest-neighbor sites on the fcc lattice are energetically unfavorable. Fast double jumps of platinum atoms are in effect jumps along the edge of the cubic elementary cell and prevent these unfavorable configurations. These jumps are called effective jumps.

It is quite impressive that by means of these simulations a quite common phenomena which we used in the past to explain the puzzling diffusion process in ordered Fe-Al [51, 25], Ni-Ga [27] or Co$\mathrm{Ga}$ [28] B2 structured alloys can be demonstrated. As explained in these previous works the vacancy is allowed to exchange with nearest-neighbor atoms exclusively but with additional interaction between the vacancy and the atoms. In such cases a perfect agreement between simulation and experiment was obtained as shown by Weinkamer et al. [50]. It was assumed that the remarkable features of diffusion in B2-structures arise from highly correlated vacancy paths, which are due to an interaction of the vacancy with locally disordered areas in the B2 lattice [50]. The Monte Carlo simulation presented here confirms this explanation to be valid also in fcc structures with short-range order. By addition of pair-interaction coefficients with further atoms or with vacancies, arbitrary diffusion paths can be created. The real challenge is conducting the experiments which shall provide results with sufficiently high statistical quality to show these diffusion paths. Carefully conducted aXPCS experiments promise to be precisely the right method for this task.

Note that the influence of SRO on the correlation times of autocorrelation functions were already demonstrated in the first experiment on $\mathrm{Cu}_{90} \mathrm{Au}_{10}$ solid solution by Leitner et al. [14]. However, only one selected alloy composition was measured. Concerns were expressed in Leitner and Vogl [13], whether the theory of Eq. 20 can be successfully applied to concentrated solid solutions. With the Monte Carlo method applied here, it is shown that the simulated chemical diffusion coefficient complies with the values calculated from the aXPCS measurements for all compositions, even in the case of strong SRO-interaction.

Parallel to the simulation of the diffuse intensities and correlation functions, also the positions of all the atoms were obtained. They were used to calculate the chemical diffusion coefficient using Eq. 35 as well as self-diffusion coefficients of both alloy components with the usual Einstein expression for the tracer diffusion Eq. 33 (in this case one uses an ensemble average $\left\langle\Delta \mathbf{r}_{X}^{2}\right\rangle$ in Eq. 33, where $\Delta \mathbf{r}_{X}$ is the displacement of an A or B atom in time $t$ ). Notice, that one shall not expect large differences between the chemical and tracer coefficients because they are simulated in a disordered fcc structure. These differences are minor even in a well ordered $\mathrm{L}_{2}$ structure as shown by Belova and Murch [44].

Special attention should be given to the comparison of Monte Carlo-simulated chemical diffusion coefficients $\tilde{D}_{\mathrm{MC}}$ with diffusion coefficient $D_{\mathrm{MC}}^{\mathrm{fit}}$ in Table $1 . D_{\mathrm{MC}}^{\mathrm{fit}}$ was calculated by fitting the inverse correlation time $\tau(\mathbf{q})^{-1}$ in Eq. 20 shown in the lower row of Fig. 5. The $\tilde{D}_{\mathrm{MC}}$ values show a good agreement with $D_{\mathrm{MC}}^{\mathrm{fit}}$ for Pt content $\lesssim 10$ at.\%. Neglecting the thermodynamic factor in the $c=20$ at.\% alloy seems to be inaccurate. In order for the modeled diffusivity to agree with that from the computer experiment it is essential to include an admixture of further jumps. In such a case the numerator of Eq. 33 becomes a sum of the different jump lengths which correspond to a sum of partial diffusion coefficients $[23,36]$. 
Table 1: Result of the Monte Carlo simulation of tracer diffusion coefficients $D_{\mathrm{Pt}}^{*}, D_{\mathrm{Ni}}^{*}$, the chemical diffusion coefficient $\tilde{D}$, jump frequency $\tau_{0}^{-1}$, contribution of $\langle 100\rangle$ jumps $\bar{p}_{100}$ and the diffusion coefficient calculated according to Eq. $33 D_{\mathrm{MC}}^{\text {fit }}$. All values were multiplied by $10^{2}$, time units are MC steps.

\begin{tabular}{l|c|c|c|c|c|r}
$c_{\mathrm{Pt}}$ [at.\%] & $D_{\mathrm{Pt}}^{*}$ & $D_{\mathrm{Ni}}^{*}$ & $\tilde{D}_{\mathrm{MC}}$ & $\tau_{0}^{-1}$ & $\bar{p}_{100}$ & $D_{\mathrm{MC}}^{\mathrm{fit}}$ \\
\hline \hline 3 & $6.1591(3)$ & $6.15737(2)$ & $6.14(1)$ & $16.4(2)$ & 0 & $5.47(7)$ \\
10 & $5.70277(1)$ & $5.7032(2)$ & $5.70(1)$ & $19.2(2)$ & 0.05 & $6.75(7)$ \\
20 & $5.6171(1)$ & $5.61855(3)$ & $5.58(1)$ & $21.7(2)$ & 0.20 & $8.71(7)$
\end{tabular}

It is well known that each method of diffusion studies has its own time scale and that the temporal resolution does not need to be in the order of single atomic jumps in order to measure correct diffusion coefficients. The same, of course, holds for aXPCS. Moreover, even in a case where effective jumps rather than single atomic jumps are observed, the underlying atomistic diffusion mechanism is accessible.

\section{Nonmetallic systems}

While metals can usually be treated in scope of a Bravais lattice, this is not the case for other materials, like semiconductors. This section is an example for a practical approach to Section Theoretical background.

Silicon Germanium. Here we present the preliminary data of aXPCS measuring a multi-exponen-
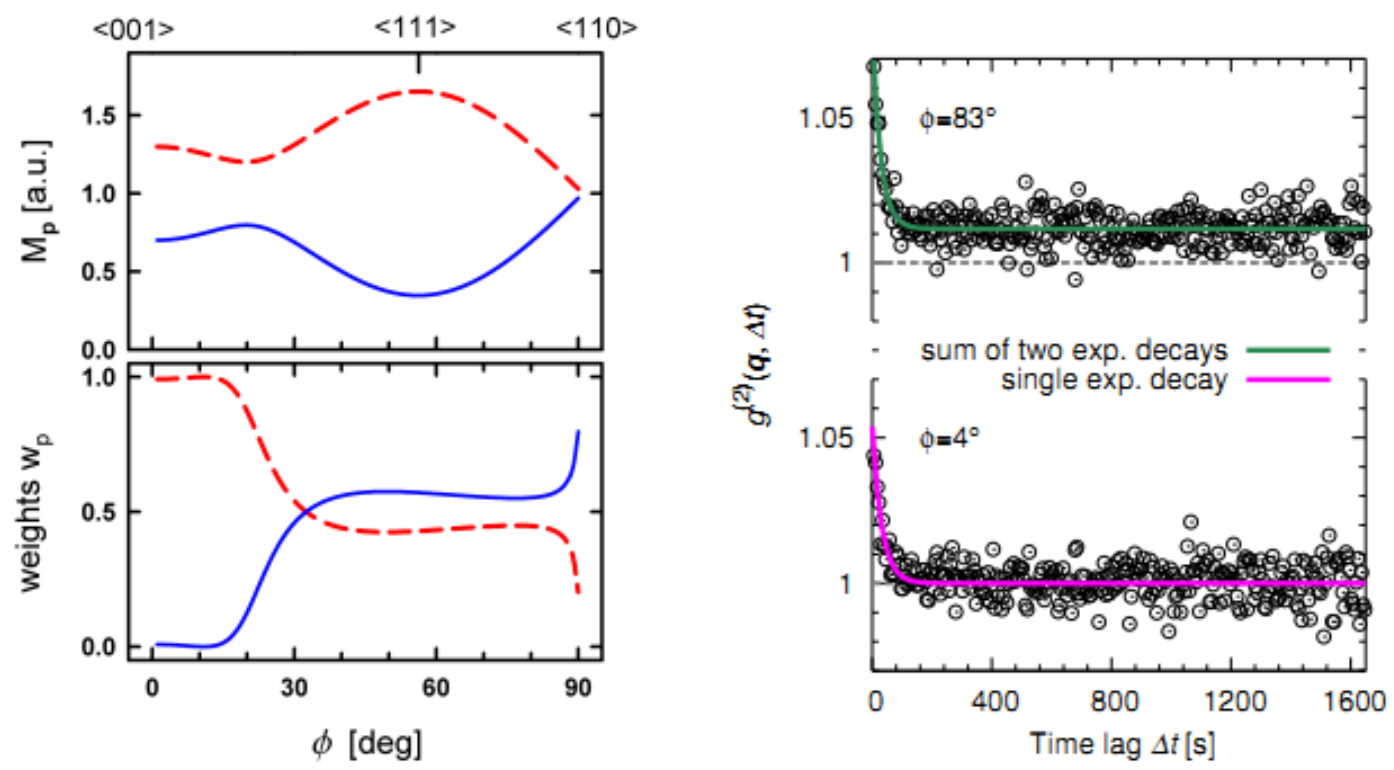

Fig. 6: Left: Theoretical prediction for the time-decay constants $\mathbf{M}_{p}$ and weights $w_{p}$ of the two processes yielding a slow (blue, solid) and a fast (red, dashed) exponential decay. These processes compose the autocorrelation function in a diamond structure with a vacancy jump mechanism of diffusion. Right: Autocorrelation function measured at $\mathrm{T}=1173 \mathrm{~K}$ in $\mathrm{Si}_{89} \mathrm{Ge}_{11}$ single crystal at $\phi=4^{\circ}$ (lower spectrum) and $\phi=83^{\circ}$ (upper spectrum) and under a scattering angle $2 \theta=20^{\circ}$.

tial autocorrelation function decay on a system unavailable for other resonant methods. The measurement was carried out on a $\mathrm{Si}_{89} \mathrm{Ge}_{11}$ single crystal. A Si-Ge mixed crystal is an ideal system for the study of germanium diffusion which is in this case a perfect substitute for silicon self-diffusion. This is exceptionally possible in this system due to the lack of chemical SRO and clustering, as was proved 
by neutron scattering experiments [52]. A $2 \times 2$ jump matrix calculated according to Eq. 26 from Section Theoretical background for the case of diffusion via a vacancy mechanism in a diamond structure has a very simple form (the matrix is Hermitian)

$$
\tilde{\mathbf{A}}=\tau_{0}^{-1}\left(\begin{array}{cc}
-1 & \eta \\
\eta^{*} & -1
\end{array}\right)
$$

where the imaginary factor $\eta$ is given as $\eta=\cos \left(\frac{a}{4} q_{x}\right) \cos \left(\frac{a}{4} q_{y}\right) \cos \left(\frac{a}{4} q_{z}\right)-\mathrm{i} \sin \left(\frac{a}{4} q_{x}\right) \sin \left(\frac{a}{4} q_{y}\right) \sin \left(\frac{a}{4} q_{z}\right)$ and $q_{x}, q_{y}, q_{z}$ are the components of the wave vector $\mathbf{q}$ along Cartesian crystal axes. The results of calculating the decay rates and weights of exponent components are shown in Fig. 6. An analytical simulation was performed for the wave vector lying close to the $(\overline{1} 10)$ crystalline plane and rotated around the [110] direction. For the details of the experiment see Section Experimental setup.

The second part of Fig. 6 shows autocorrelation functions measured at $1173 \mathrm{~K}$ in the $q$ directions corresponding to the azimuthal angles $\phi=4^{\circ}$ and $\phi=83^{\circ}$. From the simulation one can see that there are two exponential decays with the weighting factors fitting the simulation results from Fig. 6 . The autocorrelation function measured close to the [001] crystal direction appears to only consist of one exponential decay because the slow process has a weight of zero, see Fig. 6 left. The autocorrelation function measured close to the [110] direction, in contrast, appears to consist of two exponential decays. These two spectra are a hint towards a vacancy diffusion mechanism for Ge atoms diffusion in Si lattice because only this mechanism results in two decay components of the function, see Eq. 27. Other conceivable mechanisms like self-interstitial or interstitialcy mechanisms should result in only one single exponential decay of the autocorrelation function. Recent studies of the self-diffusion mechanism in isotopic ${ }^{28} \mathrm{Si} /{ }^{30} \mathrm{Si}$ multilayer by Raman spectroscopy by Shimizu et al. [53] show that the vacancy mechanism dominates up to roughly $1200 \mathrm{~K}$ and the self-interstitial above this temperature. The preliminary data indicate that the Ge impurities in the Si lattice show similar behavior.

\section{Conclusion}

The central aim of this review was to demonstrate the potential of the new method of atomic-scale Xray Photon Correlation Spectroscopy (aXPCS) for determining elementary diffusion jump processes. On the contrary to the previous methods which were limited to special isotopes and to fast diffusion processes at high temperatures, aXPCS can work in almost any substance that is composed of two or more components. It works in non-metallic systems, as well as in amorphous or polycrystalline materials. Experimental results presented in this review confirm this claim. Moreover, Monte Carlo simulations show that the theoretical foundations of aXPCS are well fulfilled also for highly concentrated solid solutions which was previously unclear.

Concluding, the future of aXPCS is promising. With new synchrotron sources of unattainable brilliance like the new XFEL source in Hamburg, combined with progresses in detector technology, fast and very fast processes and thereby a whole new range of atomic migration mechanisms will be accessible.

\section{Acknowledgments}

This research was funded by the Austrian Science Fund (FWF) contract P-22402.

Special thanks go to our collaborator Michael Leitner, now at Technische Universitat unchen for solving various theoretical problems of the XPCS method. We thank Yuriy Chushkin, Federico Zontone and the whole team of beamline ID10 at the ESRF in Grenoble, as well as Michael Sprung and the whole team of beamline P10 at PETRA III in Hamburg for their support during beam times. We thank Nikolay Abrosimov, Leibniz-Institut für Kristallzüchtung, Berlin, for providing the Si-Ge sample. We also thank Herwig Peterlik and Stephan Puchegger from the Faculty Center for Nano Structure Research of the University of Vienna for the SAXS and EDX analysis. 


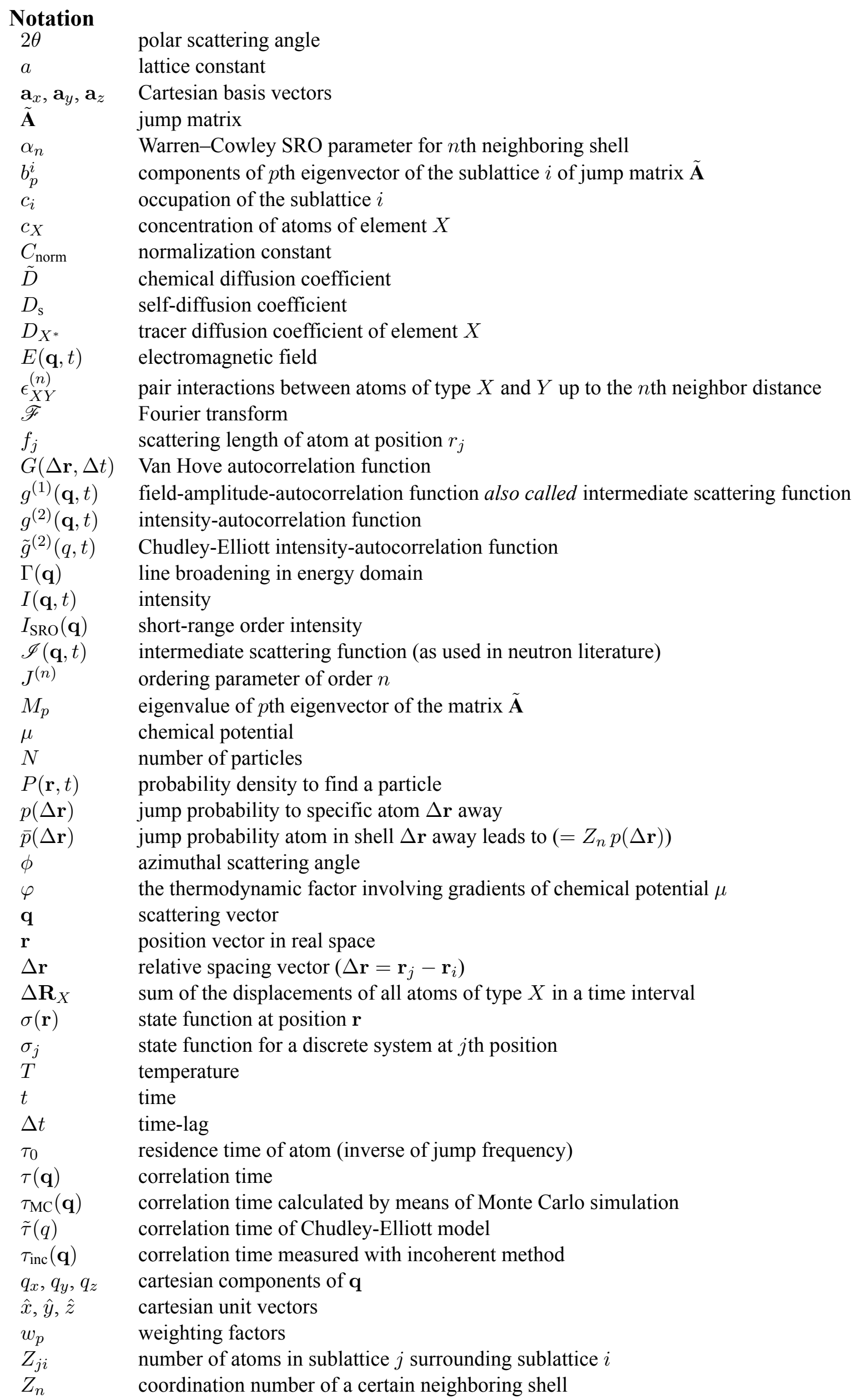


aXPCS atomic-scale X-ray photon correlation spectroscopy

CCD charge-coupled device

MC Monte Carlo

QEMS quasi-elastic Mössbauer spectroscopy

QENS quasi-elastic neutron spectroscopy

SRO short-range order

XPCS X-ray photon correlation spectroscopy

\section{References}

[1] L van Hove. Correlations in space and time and Born approximation scattering in systems of interacting particles. Physical Review, 95(1):249, 1954.

[2] R Hempelmann. Quasielastic neutron scattering and solid state diffusion. Oxford University Press, Oxford, 2000.

[3] J V Michalowicz, J M Nichols, F Bucholtz, and C C Olson. An Isserlis theorem for mixed Gaussian variables: application to the auto-bispectral density. Journal of Statistical Physics, 136(1):89--102, 2009.

[4] P A Lemieux and D J Durian. Investigating non-Gaussian scattering processes by using $n$ th-order intensity correlation functions. J. Opt. Soc. Am., 16(7):1651--1664, 1999.

[5] A S Gittings and D J Durian. Gaussian and non-Gaussian speckle fluctuations in the diffusingwave spectroscopy signal of a coarsening foam. Appl. Opt., 45(10):2199--2204, Apr 2006.

[6] R Pecora. Dynamic Light Scattering: AQpplications of Photon Correlation Spectroscopy. Springer, 1985.

[7] L Cipelletti and E C Weeks. Glassy dynamics and dynamical heterogeneity in colloids. In L. Berthier, G. Biroli, J.-P. Bouchaud, L. Cipelletti, and W. van Saarlos, editors, Dynamical Heterogeneities in Glasses, Colloids and Granular Media, pages 110--151. Oxford University Press, 2011.

[8] J W Haus and K W Kehr. Diffusion in regular and disordered lattices. Physics Reports-Review Section of Physics Letters, 150(5-6):263--406, JUN 1987.

[9] M Sutton. X-ray intensity fluctuation spectroscopy. In F Hippert, E Geissler, J-L Hodeau, E Lelievre-Berna, and J-R Regnard, editors, Neutron and X-ray Spectroscopy, pages 297--318. Springer Netherlands, 2006.

[10] G Vogl and B Sepiol. The elementary diffusion step in metals studied by the interefrence of gamma-rays, X-rays and neutrons. In P. Heitjans and J. Kärger, editors, Diffusion in Condensed Matter, pages 65--91. Springer, 2005.

[11] P G De Gennes. Liquid dynamics and inelastic scattering of neutrons. Physica, 25(7-12):825-$839,1959$.

[12] S K Sinha and D K Ross. Self-consistent density response function method for dynamics of light interstitials in crystals. Physica $B+C, 149(1-3): 51$-- 56, 1988.

[13] M Leitner and G Vogl. Quasi-elastic scattering under short-range order: the linear regime and beyond. J. Phys.-Condens. Mat., 23:254206, 2011. 
[14] M Leitner, B Sepiol, L-M Stadler, B Pfau, and G Vogl. Atomic diffusion studied with coherent X-rays. Nature Materials, 8(9):717--720, 2009.

[15] M Stana, M Leitner, M Ross, and M Sepiol. Studies of atomic diffusion in Ni-Pt solid solution by X-ray photon correlation spectroscopy. Journal of Physics: Condensed Matter, 25(6):065401, 2013.

[16] M Ross, M Stana, M Leitner, and B Sepiol. Direct observation of atomic network migration in glass. New J. Phys. in press.

[17] C T Chudley and R J Elliott. Neutron scattering from a liquid on a jump diffusion model. Proc. Phys. Soc., 77:353, 1961.

[18] G Sauthoff. Intermetallics. Wiley-VCH Verlag GmbH, 2007.

[19] R Kutner and I Sosnowska. Thermal neutron scattering from a hydrogen-metal system in terms of a general multi-sublattice jump diffusion model I: Theory. Journal of Physics and Chemistry of Solids, 38(7):741 -- 746, 1977.

[20] J M Rowe, K Skoeld, H E Flotow, and J J Rush. Quasielastic neutron scattering by hydrogen in the alpha and beta phases of vanadium hydride. Journal of Physics and Chemistry of Solids, 32(1):41 -- 54, 1971.

[21] I S Anderson, A Heidemann, J E Bonnet, D K Ross, S K P Wilson, and M W McKergow. Proton residence times in the solid solution phase of the Y--H system studied by quasi-elastic neutron scattering. Journal of the Less Common Metals, 101:405--418, 1984.

[22] O G Randl, B Sepiol, G Vogl, R Feldwisch, and K Schroeder. Quasielastic Mössbauer spectroscopy and quasielastic neutron scattering from non-Bravais lattices with differently occupied sublattices. Phys. Rev. B, 49:8768--8773, 1994.

[23] B Sepiol and K F Ludwig. High-resolution experimental methods. In W Pfeiler, editor, Alloy Physics: A Comprehensive Reference, pages 707--773. Wiley-VCH, 2007.

[24] B Sepiol and G Vogl. Atomistic determination of diffusion mechanism on an ordered lattice. Phys. Rev. Lett., 71(5):731--734, Aug 1993.

[25] R Feldwisch, B Sepiol, and G Vogl. Elementary diffusion jump of iron atoms in intermetallic phases studied by Mössbauer spectroscopy - II. from order to disorder. Acta metall. mater., 43(5):2033--2039, 1995.

[26] B Sepiol, A Meyer, G Vogl, R Ruffer, A I Chumakov, and A Q R Baron. Time domain study of Fe-57 diffusion using nuclear forward scattering of synchrotron radiation. Phys. Rev. Lett., 76(17):3220--3223, APR 221996.

[27] M Kaisermayr, J Combet, H Ipser, H Schicketanz, B Sepiol, and G Vogl. Nickel diffusion in B2-NiGa studied with quasielastic neutron scattering. Phys. Rev. B, 61(18):12038--12044, 2000.

[28] M Kaisermayr, J Combet, H Ipser, H Schicketanz, B Sepiol, and G Vogl. Determination of the elementary jump of Co in CoGa by quasielastic neutron scattering. Phys. Rev. B, 63:054303, Jan 2001 .

[29] M Kaisermayr, C Pappas, B Sepiol, and G Vogl. Probing jump diffusion in crystalline solids with neutron spin-echo spectroscopy. Phys. Rev. Lett., 87(17), 2001. 
[30] M Kaisermayr, M Rennhofer, G Vogl, C Pappas, and S Longeville. Neutron spin-echo spectroscopy for diffusion in crystalline solids. Phys. Rev. B, 66:024302, Jun 2002.

[31] T Salditt, S Kalbfleisch, M Osterhoff, S P Krüger, M Bartels, K Giewekemeyer, H Neubauer, and M Sprung. Partially coherent nano-focused x-ray radiation characterized by Talbot interferometry. Optics Express, 19(10):9656--9675, 2011.

[32] M Leitner. Studying Atomic Dynamics with Coherent X-rays. Springer, 2012.

[33] F Livet, F Bley, J Mainville, R Caudron, S G J Mochrie, E Geissler, G Dolino, D Abernathy, $\mathrm{G}$ Grübel, and M Sutton. Using direct illumination CCDs as high-resolution area detectors for X-ray scattering. Nucl. Instrum. Meth. A, 451(3):596--609, 2000.

[34] D Lumma, L B Lurio, S G J Mochrie, and M Sutton. Area detector based photon correlation in the regime of short data batches: Data reduction for dynamic X-ray scattering. Review of Scientific Instruments, 71(9):3274--3289, 2000.

[35] R Dinapoli, A Bergamaschi, D Greiffenberg, B Henrich, R Horisberger, I Johnson, A Mozzanica, V Radicci, B Schmitt, X Shi, et al. EIGER characterization results. Nuclear Instruments and Methods in Physics Research Section A: Accelerators, Spectrometers, Detectors and Associated Equipment, 2013.

[36] J Philibert. Atom Movements Diffusion and Mass Transport in Solids, translated from the French by Steven J Rothman. Les Editions de Physique, Paris, France, 1991.

[37] H Mehrer. Diffusion in solids: fundamentals, methods, materials, diffusion-controlled processes. Springer Verlag, 2007.

[38] D Wolf. Theory of Mössbauer line broadening due to correlated diffusion in crystals. Appl. Phys. Lett., 30(12):617--619, 1977.

[39] F Haider, R Kozubski, and T A Abinandanan. Simulation Techniques. In W Pfeiler, editor, Alloy Physics: A Comprehensive Reference, pages 653--706. Wiley-VCH, 2007.

[40] J M Cowley. Short- and long-range order parameters in disordered solid solutions. Phys. Rev., 120:1648--1657, 1960.

[41] G E Murch and I V Belova. Chemical diffusion by vacancy pairs in intermetallic compounds with the B2 structure. Philosophical Magazine Letters, 80(8):569--575, 2000.

[42] L So Darken. Diffusion, mobility and their interrelation through free energy in binary metallic systems. Trans. Aime, 175(184):41, 1948.

[43] J R Manning. Diffusion kinetics in crystals. Van Norstrand, Princeton, New Jersey, page 117, 1968.

[44] I V Belova and G E Murch. Test of the validity of the Darken/Manning relation for diffusion in ordered alloys taking the L12 structure. Philosophical Magazine A, 78(5):1085--1092, 1998.

[45] M C Cadeville, C E Dahmani, and F Kern. Magnetism and spatial order in Ni-Pt and Co-Pt alloys. J. Magn. Magn. Mater., 54:1055--1056, 1986.

[46] R E Parra and J W Cable. Neutron study of magnetic-moment distribution in Ni-Pt alloys. Phys. Rev. B, 21:5494--5504, 1980. 
[47] R E Parra, R Medina, and J W Cable. Determination of lattice distortions in concentrated alloys by neutron diffuse scattering. Solid State Communications, 40(6):679--682, 1981.

[48] B E Warren, B L Averbach, and B W Roberts. Atomic Size Effect in the X-Ray Scattering by Alloys. Journal of Applied Physics, 22(12):1493--1496, 1951.

[49] M Leitner and R Podloucky. Preliminary ab initio calculations of the interaction constants in Ni-Pt alloys. private communication, 2013.

[50] R Weinkamer, P Fratzl, B Sepiol, and G Vogl. Monte Carlo simulations of Mössbauer spectra in diffusion investigations. Phys. Rev. B, 59:8622--8625, 1999.

[51] R Feldwisch, B Sepiol, and G Vogl. Elementary diffusion jump of iron atoms in intermetallic phases studied by Mössbauer spectroscopy - I. Fe-Al close to equiatomic stoichiometry. Acta metall. mater., 42(9):3175 -- 3181, 1994.

[52] D Le Bolloc'h, J L Robertson, H Reichert, S C Moss, and M L Crow. X-ray and neutron scattering study of Si-rich Si-Ge single crystals. Phys. Rev. B, 63:035204, Jan 2001.

[53] Y Shimizu, M Uematsu, and K M Itoh. Experimental evidence of the vacancy-mediated silicon self-diffusion in single-crystalline silicon. Phys. Rev. Lett., 98:095901, Mar 2007. 